\title{
(2) OPEN ACCESS \\ Development and validation of a predictive algorithm for risk of dementia in the community setting
}

\author{
Stacey Fisher (1) 1,2,3 Douglas G Manuel (1) 1,2,3,4,5 Amy T Hsu, 1,2,3,5 Carol Bennett, ${ }^{1,2}$ \\ Meltem Tuna, ${ }^{1,2}$ Anan Bader Eddeen, ${ }^{1,2}$ Yulric Sequeira, ${ }_{1}^{1,3}$ Mahsa Jessri, ${ }^{1,2,4}$ \\ Monica Taljaard, ${ }^{1,3}$ Geoffrey M Anderson, ${ }^{6,7}$ Peter Tanuseputro ${ }^{1,2,5,8}$
}

\begin{abstract}
- Additional material is published online only. To view, please visit the journal online (http://dx.doi.org/10.1136/ jech-2020-214797).

${ }^{1}$ Clinical Epidemiology Program, Ottawa Hospital Research Institute, Ottawa, Ontario, Canada

${ }^{2}$ Populations \& Public Health, ICES, Ottawa, Ontario, Canada ${ }^{3}$ School of Epidemiology and Public Health, University of Ottawa, Ottawa, Ontario, Canada

${ }^{4}$ Health Analysis Division, Statistics Canada, Ottawa, Ontario, Canada ${ }^{5}$ Centre for Individualized Health, Bruyere Research Institute, Ottawa, Ontario, Canada

${ }^{6}$ Cardiovascular Research, ICES, Toronto, Ontario, Canada ${ }^{7}$ Institute of Health Policy, Management and Evaluation, University of Toronto, Toronto, Ontario, Canada

${ }^{8}$ Department of Medicine, University of Ottawa, Ottawa, ON, Canada
\end{abstract}

\section{Correspondence to}

Dr Stacey Fisher, Dalla Lana School of Public Health, University of Toronto, Toronto, ON M5T 3M7, Canada; stacey.fisher@utoronto.ca

Received 11 June 2020 Revised 30 October 2020 Accepted 4 November 2020 Published Online First 24 June 2021

\begin{abstract}
Background Most dementia algorithms are unsuitable for population-level assessment and planning as they are designed for use in the clinical setting. A predictive risk algorithm to estimate 5-year dementia risk in the community setting was developed.

Methods The Dementia Population Risk Tool (DemPoRT) was derived using Ontario respondents to the Canadian Community Health Survey (survey years 2001 to 2012). Five-year incidence of physiciandiagnosed dementia was ascertained by individual linkage to administrative healthcare databases and using a validated case ascertainment definition with followup to March 2017. Sex-specific proportional hazards regression models considering competing risk of death were developed using self-reported risk factors including information on socio-demographic characteristics, general and chronic health conditions, health behaviours and physical function.
\end{abstract}

Results Among 75460 respondents included in the combined derivation and validation cohorts, there were 8448 cases of incident dementia in 348677 personyears of follow-up (5-year cumulative incidence, men: 0.044, 95\% Cl: 0.042 to 0.047; women: $0.057,95 \% \mathrm{Cl}$ : 0.055 to 0.060). The final full models each include $90 \mathrm{df}$ (65 main effects and 25 interactions) and 28 predictors (8 continuous). The DemPoRT algorithm is discriminating (C-statistic in validation data: men 0.83 (95\% Cl: 0.81 to 0.85$)$; women 0.83 (95\% Cl: 0.81 to 0.85$)$ ) and well-calibrated in a wide range of subgroups including behavioural risk exposure categories, socio-demographic groups and by diabetes and hypertension status.

Conclusions This algorithm will support the development and evaluation of population-level dementia prevention strategies, support decision-making for population health and can be used by individuals or their clinicians for individual risk assessment.

\section{INTRODUCTION}

An estimated 50 million people worldwide have dementia, which is expected to grow to over 152 million by $2050,{ }^{1}$ putting a tremendous strain on our healthcare systems, caregivers and families. As there is no cure or disease-modifying therapies and an estimated $30 \%$ of dementia may be attributable to potentially modifiable factors, ${ }^{2}$ primary prevention has become an important component of healthcare planning and policy development and has been identified as a primary objective of many international dementia strategies. ${ }^{3-6}$ Population risk prediction algorithms can be used to describe population dementia risk, project the number of new cases over time and inform the development of prevention strategies by identifying optimal target groups for intervention and estimating the potential population health benefit. ${ }^{7}$

Most existing dementia algorithms are designed for use in the clinical setting and are not suitable for population health planning purposes. For a population risk algorithm, input variables need to be available to population health planners and decision makers, representative of the population and regularly collected so estimates can be frequently updated. Many existing dementia algorithms include neuropsychological assessment, ${ }^{8-13}$ genetic testing, ${ }^{10-14}$ neuroimaging ${ }^{10}$ or other clinical variables (eg, blood pressure or cholesterol values) ${ }^{14-17}$ that do not usually fulfil these criteria. Additionally, population algorithms should be developed on large, representative data sets and include a wide range of socio-demographic variables to allow for risk assessment and equity evaluation across population health subgroups including ethnicity, education and immigrant status. Lastly, the inclusion of modifiable risk factors facilitates the evaluation of preventative strategies. Few existing dementia algorithms are suited for population health planning.

The objective of this study was to develop and validate a risk algorithm for dementia incidence in the community setting using population health survey data. The Dementia Population Risk Tool (DemPoRT) can be used to inform the development of dementia prevention strategies and support decision-making for population health. In addition to this population health planning purpose, DemPoRT can also be used by patients or their clinicians to assess individual dementia risk.

\section{METHODS}

\section{Study design and participants}

This prospective cohort study used population health survey data linked to health administrative dementia data to develop and validate a population risk algorithm, DemPoRT, for predicting 5-year dementia incidence in the community setting. Dementia was ascertained using physician billing, hospitalisation and drug dispensing data with follow-up to March 2017. Model development can be summarised in to four steps:

1. Model derivation - creation of male and female DemPoRT risk algorithms using respondents to 
the 2001, 2003, 2005 and 2007/2008 Canadian Community Health Surveys (CCHS);

2. Model validation - validation of the DemPoRT algorithms using the 2009/2010 and 2011/2012 CCHS;

3. Final model generation - estimation of final, full DemPoRT models using the combined derivation and validation data and the same model specification as the derivation models;

4. Derivation of the application model - creation of a parsimonious model with fewer predictors that attempts to maintain discriminatory ability, calibration and overall model performance.

The protocol for development and validation of DemPoRT was registered and published (ClinicalTrials.gov, NCT03155815). ${ }^{18}$ We adhered to the protocol with the following exceptions: the validated dementia definition was not supplemented with dementia information from home care and long-term care data, as the data is not available prior to 2008; individuals had to be age 55 or older at the time of survey administration; follow-up was extended to March 2017 with the availability of new data; ethnicity and functional measures were recategorised due to small sample sizes; variables for multilingualism, chronic obstructive pulmonary disorder and epilepsy were added; and only the first of the multiply imputed data sets was used for model analyses, informed by previous work with this data. ${ }^{19}$ This paper adheres to the Transparent Reporting of a multivariable prediction model for Individual Prognosis or Diagnosis (TRIPOD) checklist for prediction model development. ${ }^{20}$

Survey respondents who agreed to share and link their survey interview information were eligible for study inclusion. Respondents were excluded if they were not eligible for Ontario's universal health insurance programme, indicated a diagnosis of dementia in the CCHS or were younger than age 55 at the time of survey administration. For individuals with multiple CCHS interviews, only the earliest interview was included.

\section{Data sources}

The CCHS is a national, cross-sectional survey developed by Statistics Canada to collect data related to health determinants, health status and healthcare use. It employs a complex multistage sampling strategy to randomly select households in each region, with a target population of individuals aged 12 years and older. Over the study period, the surveys attained an average response rate of 79\%. Individuals living on First Nation Reserves, institutionalised residents, full-time members of the Canadian Forces and residents of certain remote areas are excluded. Details of the survey methodology have been previously published. ${ }^{21}$ Model predictors were ascertained from self-reported responses to the CCHS. The derivation cohort was comprised of Ontario respondents to the CCHS conducted in 2001, 2003, 2005 and $2007 / 2008$. The validation cohort consisted of Ontario CCHS respondents from 2009/2010 and 2011/2012. Temporal validation was used as it is a stronger validation approach than random creation of development and validation data sets, and because this tool will be used for prediction of future dementia risk. ${ }^{22}$

Dementia incidence was ascertained using population-based data sets housed at ICES (formerly known as the Institute for Clinical Evaluative Sciences), which have been linked at the individual level to CCHS respondents. These data sets include hospital-admission records from the Canadian Institute for Health Information Discharge Abstract Database, physician billing and diagnoses from the Ontario Health Insurance Plan physician claims database, hospital and community-based ambulatory care from the National Ambulatory Care Reporting
System, and drug dispensing data from the Ontario Drug Benefit programme. Death was ascertained using Ontario Vital Statistics and the Registered Persons Database.

\section{Outcome}

The primary outcome of interest was 5-year incidence of physician-diagnosed dementia, ascertained using a validated case ascertainment definition: one hospital record OR three physician claims records at least 30 days apart within a 2 -year period OR a dispensing record for a cholinesterase inhibitor. ${ }^{23}$ This definition has a $79.3 \%$ sensitivity and a $99.1 \%$ specificity when validated against emergency medical record data. Survey respondents were followed from survey administration date until the earliest of dementia ascertainment, death, loss to follow-up (defined as loss of healthcare eligibility) or end of study (31 March 2017).

\section{Statistical methods and analyses}

The analysis plan was developed following guidelines by Harrell $^{24}$ and Steyerberg ${ }^{25}$ and informed by the development of other algorithms by the team. ${ }^{1926}$

Predictor identification, data cleaning, missing data and model specification methods have previously been described in the study protocol. ${ }^{18}$ Predictor variables were selected from the CCHS informed by review of existing dementia prediction algorithms, ${ }^{27}$ subject-matter expertise, our previous work developing population risk algorithms using this data ${ }^{19} 26$ and variable availability across cycles. Predictor identification and specification as well as all data cleaning and coding occurred prior to examining exposure-outcome associations. Preliminary sex-specific main effects models were fit using the prespecified predictors and degree of freedom (df) allocation. Partial association $\chi^{2}$ statistics for each predictor minus their $\mathrm{df}$ were plotted to inform df reduction, however, all initial $\mathrm{df}$ were retained.

Table 1 presents the 33 prespecified predictor variables and the final 29 variables in the full model, after recategorisation of ethnicity and functional measures. The models include interaction between age and all variables except socio-demographic, general health and survey-year variables, with continuous variable interactions restricted to linear terms. Survey questions used to ascertain the model variables are available at https:/github. com/Big-Life-Lab/DemPoRT.

Models were estimated using Fine and Gray subdistribution hazard models, ${ }^{28}$ considering death as a competing risk. Sexspecific models were created as the effect of many dementia risk factors varies by sex, and as modelling with interaction terms would be difficult considering that age interaction is also being included for many of the predictor variables. All predictors were centred on their means for ease of recalibration in new populations and to allow for application in individuals or settings with missing values. Plots of raw and smoothed scaled Schoenfeld residuals versus time for each predictor were assessed. Overfitting was assessed in the full models using the heuristic shrinkage estimator, ${ }^{29}$ which indicated that shrinkage was unnecessary $(m e n=0.97$; women $=0.98)$. Due to known challenges using survey weights in regression modelling, ${ }^{30}$ survey weights were not used for model derivation, however we recommend their use for population application and reporting. ${ }^{26}$ We used a step-down procedure described by Ambler $^{31}$ to create a smaller, reduced model from the full model. This procedure involves removing variables that result in the smallest decrease in model $\mathrm{R}^{2}$, one variable at a time, until the Akaike Information Criterion is minimised. Two sensitivity analyses were performed using the combined data to explore model performance: (1) if the number 
Table 1 Predictor variables for the Dementia Population Risk Tool (DemPoRT) models

\begin{tabular}{|c|c|c|c|c|}
\hline Variable & Scale & Initial variable specification & Full model & Reduced model \\
\hline \multicolumn{5}{|l|}{ df } \\
\hline Male model & - & 88 (65 main, 23 interaction) & 90 (65 main, 25 interaction) & 74 (51 main, 23 interaction) \\
\hline \multicolumn{5}{|l|}{ Socio-demographic factors } \\
\hline Age $^{*}$ & Continuous & Five knot spline: Valid range: 55-102 (male), 55-101 (female) & Unchanged & Unchanged \\
\hline Sex & Categorical & Stratified: Male; female & Unchanged & Unchanged \\
\hline Immigrant & Dichotomous & Yes; no & Unchanged & Excluded \\
\hline Education & Categorical & $\begin{array}{l}\text { Four categories: Less than secondary school; secondary school } \\
\text { graduation; some post-secondary; post-secondary graduation }\end{array}$ & Unchanged & Excluded (male) \\
\hline Multilingualism & Dichotomous & - & Multilingual; not multilingual & Excluded (female) \\
\hline \multicolumn{5}{|l|}{ General health } \\
\hline Sense of belonging & Ordinal & $\begin{array}{l}\text { Four categories: Very strong; somewhat strong; somewhat weak; } \\
\text { very weak }\end{array}$ & Unchanged & Excluded \\
\hline Stress & Ordinal & $\begin{array}{l}\text { Five categories: Not at all stressful; not very stressful; a bit } \\
\text { stressful; quite a bit stressful; extremely stressful }\end{array}$ & Unchanged & Unchanged \\
\hline Self-rated health & Ordinal & Five categories: Poor; fair; good; very good; excellent & Unchanged & Excluded (female) \\
\hline \multicolumn{5}{|l|}{ Health behaviours } \\
\hline Smoking status & Categorical & $\begin{array}{l}\text { Four categories: Non-smoker; current smoker; former smoker quit } \\
<5 \text { years ago; former smoker quit } \geq 5 \text { years ago }\end{array}$ & Unchanged & Unchanged \\
\hline Juice consumption & Continuous & Three knot spline: Valid range: $0-6$ (male), $0-5$ (female) & Unchanged & Unchanged \\
\hline $\begin{array}{l}\text { Leisure physical } \\
\text { activity } \\
\text { (METs) }\end{array}$ & Continuous & Three knot spline: Valid range: 0-16 (male), 0-12 (female) & Unchanged & Unchanged \\
\hline \multicolumn{5}{|l|}{ Functional measures } \\
\hline $\begin{array}{l}\text { Personal hygiene and } \\
\text { care }\end{array}$ & Dichotomous & Does not need help; needs help & Excluded & - \\
\hline $\begin{array}{l}\text { Locomotion in the } \\
\text { home }\end{array}$ & Dichotomous & Does not need help; needs help & Excluded & - \\
\hline Meal preparation & Dichotomous & Does not need help; needs help & Excluded & - \\
\hline Running errands & Dichotomous & Does not need help; needs help & Excluded & - \\
\hline Ordinary housework & Dichotomous & Does not need help; needs help & Excluded & - \\
\hline Heavy housework & Dichotomous & Does not need help; needs help & Excluded & - \\
\hline Finances & Dichotomous & Does not need help; needs help & Excluded & - \\
\hline $\begin{array}{l}\text { Number of activities } \\
\text { needing help }\end{array}$ & Categorical & - & $\begin{array}{l}\text { Seven categories: None; } 1 ; 2 ; 3 \\
4 ; 5 ; 6 \text { (sum of above measures, } \\
\text { except heavy housework) }\end{array}$ & Unchanged \\
\hline \multicolumn{5}{|l|}{ Health conditions } \\
\hline Heart disease & Dichotomous & Yes; no & Unchanged & Unchanged \\
\hline Stroke & Dichotomous & Yes; no & Unchanged & Unchanged \\
\hline Diabetes & Dichotomous & Yes; no & Unchanged & Unchanged \\
\hline
\end{tabular}




\begin{tabular}{lllll}
\hline \multicolumn{2}{l}{ Table 1 Continued } & & & \\
\hline Variable & Scale & Initial variable specification & Full model & Reduced model \\
\hline Mood disorder & Dichotomous & Yes; no & Unchanged & Unchanged \\
\hline High blood pressure & Dichotomous & Yes; no & Unchanged & Unchanged \\
\hline COPD & Dichotomous & - & Yes; no & Unchanged \\
Epilepsy & Dichotomous & - & Yes; no & Excluded (male) \\
Body mass index & Continuous & Threeknot spline: Valid range: 10-44 (male), 10-47 (female) & Unchanged & Unchanged \\
Design & & & & Unchanged \\
\multicolumn{2}{c}{ Survey year } & Ordinal & Six categories: 2001, 2003, 2005, 2007/2008, 2009/2010, & Unchanged \\
\hline
\end{tabular}

${ }^{*}$ Age interaction included for all variables except socio-demographic, general health and survey-year variables.

COPD, chronic obstructive pulmonary disease; $\mathrm{df}$, degrees of freedom; MET, metabolic equivalent of task.

of daily activities for which help is needed and self-rated health variables were excluded, and; (2) with age as the only predictor.

Model performance was assessed using overall measures of predictive accuracy, discrimination (how well the model is able to separate those who experience the outcome from those who do not) and calibration (agreement between predicted and observed risk). Predictive accuracy was assessed with Nagelkerke's $\mathrm{R}^{2} 32$ and the scaled Brier score. ${ }^{33}$ Discrimination was assessed using Harrell's concordance statistic (c-statistic). ${ }^{24}$ Overall calibration, calibration within deciles of predicted risk and within subgroups of importance to clinicians and policymakers were assessed. Calibration within subgroups was evaluated using a predefined standard, defined as less than a $20 \%$ difference between the observed and predicted risk estimates in subgroups where at least $5 \%$ of individuals developed dementia. ${ }^{19} 26$

Analyses were conducted in R V.3.1 ${ }^{34}$ using the riskRegres$\operatorname{sion}^{35}$ and $\mathrm{Hmisc}^{36}$ packages.

\section{Ethics approval}

ICES is a not-for-profit research institute and a prescribed health information custodian under section 45 of Ontario's Personal Health Information Privacy Act. Projects conducted under section 45 , by definition, do not require review by a Research Ethics Board. This project was conducted under section 45 and approved by ICES' Privacy and Legal Office.

\section{RESULTS Participants}

The 2001 to 2011/2012 CCHS include 253189 Ontario respondents, of which 200320 agreed to share their file, were successfully linked to administrative data at ICES, and were eligible for Ontario's health insurance plan. Of these, 78097 were at least 55 years of age at survey administration. After exclusion of those with prevalent dementia $(n=2637)$, the derivation and validation cohorts included 47739 and 27721 respondents with 472399 (men: 10.0 median years, IQR: 7.5 to 13.4 ; women: 10.2 median years, IQR 8.2 to 13.5) and 157929 (men: 5.9 median years, IQR 4.7 to 7.0 ; women: 5.9 median years, IQR 4.8 to 7.0$)$ total person-years of follow-up, respectively. In the derivation data, during the 5 -year predicted time horizon of interest (220972 person-years of follow-up), there were 6734 dementia events and 2521 deaths without dementia; in the validation data, there were 1714 incident cases of dementia and 1354 deaths without dementia over 127705 person-years. Cumulative incidence curves for 5 -year dementia ascertainment and death are in online supplemental digital content 1 . The crude 5 -year incidence rate of dementia was 2.0 per 1000 person-years among men and 2.7 per 1000 person-years among women, in the combined derivation and validation cohorts. Mean age at dementia ascertainment among men was 80.4 years (IQR: 75.6 to 85.6) and among women was 82.6 years (IQR 77.9 to 88.1).

Characteristics of the study populations are presented in table 2, with detailed information about missing provided in online supplemental digital content 2. Mean age in the derivation cohorts was 66.0 (IQR 60.0 to 74.0) among men and 68.0 (IQR 61.0 to 76.0 ) among women and was similar in the validation cohorts. There was less than $1 \%$ missing data for most predictors. With the exception of variables that were not collected in all study years (multilingualism, former vs non-drinker, needing help with finances, mood disorder, epilepsy and chronic obstructive pulmonary disorder), smoking status had the most missing data (males: 11.2\% ; females: $11.4 \%$ ), due to missing information about time since quit among former smokers.

\section{Model specification, development and validation}

Predictor variables and $\mathrm{df}$ for the full and reduced models are presented in table 1. Partial correlation plots are available in online supplemental digital content 3 . The final full models for men and women each include a total of $90 \mathrm{df}$ (65 main effects and 25 interactions), with 28 predictors (8 continuous) and 24 interaction terms (table 1). The reduced models both have 74 df (men: 51 main and 23 interactions; women: 50 main and 24 interactions). Online supplemental digital content 4 and 5 present subdistribution HRs from the full and reduced models for men and women, respectively; an interactive online visualisation tool is in development to facilitate understanding of how the risk factors contribute to dementia risk. Model formulas and beta coefficients are available in online supplemental digital content 6 and at https:/github.com/Big-Life-Lab/DemPoRT, respectively.

\section{Model performance}

Table 3 presents summary indicators of model performance. Both the male and female models are discriminating, indicating good ability to separate those who develop dementia from those who do not (male c-statistic: 0.83 , 95\% CI: 0.81 to 0.85 ; female c-statistic: $0.83,95 \% \mathrm{CI}: 0.81$ and 0.85 in validation data). Discrimination remained stable across derivation, validation and pooled data, and in the reduced model. Within the validation data, the predicted number of dementia events somewhat differed from the observed number of events (percent difference between the 5 -year observed cumulative incidence and the predicted risk, men: 4.21\%; women: $-10.58 \%$ ), while they were very similar within the reduced models (men: $-0.61 \%$; women: $-0.78 \%$ ).

Calibration across deciles of predicted risk is presented in figure 1. Calibration slopes in the validation data were 0.7859 
Table 2 Baseline study characteristics of male and female derivation and validation cohorts

\begin{tabular}{|c|c|c|c|c|}
\hline \multirow[b]{2}{*}{ Characteristic* } & \multicolumn{2}{|l|}{ Male cohort } & \multicolumn{2}{|l|}{ Female cohort } \\
\hline & Derivationt & Validation $\ddagger$ & Derivationt & Validation‡ \\
\hline $\mathrm{N}$ & 20506 & 11791 & 27233 & 15930 \\
\hline Dementia events (5 years) & 2367 & 647 & 4367 & 1067 \\
\hline Deaths (5 years) & 905 & 518 & 1616 & 836 \\
\hline \multicolumn{5}{|l|}{ Socio-demographic factors } \\
\hline \multicolumn{5}{|l|}{ Ethnicity } \\
\hline White & $19145(93.4)$ & $10785(91.5)$ & $25700(94.4)$ & $14765(92.7)$ \\
\hline South Asian/Arab/West Asian & $347(1.7)$ & $208(1.8)$ & $360(1.3)$ & $184(1.2)$ \\
\hline Japanese/Korean/South East Asian/Filipino & $274(1.3)$ & $224(1.9)$ & $243(0.9)$ & $276(1.7)$ \\
\hline Other/multiple origin/unknown & $637(3.1)$ & $479(4.1)$ & $831(3.1)$ & $597(3.7)$ \\
\hline \multicolumn{5}{|l|}{ Education } \\
\hline Less than secondary school & $6380(31.1)$ & $2792(23.7)$ & $9402(34.5)$ & $3833(24.1)$ \\
\hline Secondary school graduate & $2902(14.2)$ & $1840(15.6)$ & $5494(20.2)$ & $3429(21.5)$ \\
\hline Some post-secondary & $1148(5.6)$ & $499(4.2)$ & $1454(5.3)$ & $665(4.2)$ \\
\hline Post-secondary graduate & $9847(48.0)$ & $6564(55.7)$ & $10636(39.1)$ & $7888(49.5)$ \\
\hline \multicolumn{5}{|l|}{ Marital status } \\
\hline Now married/common-law & $14791(72.1)$ & $8316(70.5)$ & $13070(48.0)$ & $7873(49.4)$ \\
\hline Separated/divorced & $2122(10.3)$ & $1358(11.5)$ & $3180(11.7)$ & $2130(13.4)$ \\
\hline Widowed & $2224(10.8)$ & $1175(10.0)$ & $9642(35.4)$ & 4928 (30.9) \\
\hline Single & $1364(6.7)$ & $934(7.9)$ & $1325(4.9)$ & $981(6.2)$ \\
\hline \multicolumn{5}{|l|}{ General health } \\
\hline \multicolumn{5}{|l|}{ Sense of belonging } \\
\hline Very strong & $5075(24.7)$ & $2766(23.5)$ & $7156(26.3)$ & $3987(25.0)$ \\
\hline Somewhat strong & $8951(43.7)$ & $5518(46.8)$ & $12011(44.1)$ & $7657(48.1)$ \\
\hline Somewhat weak & $3805(18.6)$ & 2075 (17.6) & $5083(18.7)$ & $2709(17.0)$ \\
\hline Very weak & $1628(7.9)$ & $859(7.3)$ & $2271(8.3)$ & $1095(6.9)$ \\
\hline \multicolumn{5}{|l|}{ Self-perceived stress } \\
\hline Not at all stressful & $4871(23.8)$ & $2616(22.2)$ & $5102(18.7)$ & $2616(16.4)$ \\
\hline Not very stressful & $6420(31.3)$ & $3635(30.8)$ & $8554(31.4)$ & $4772(30.0)$ \\
\hline A bit stressful & $6491(31.7)$ & 3867 (32.8) & $9394(34.5)$ & $5739(36.0)$ \\
\hline Quite a bit stressful & $2154(10.5)$ & $1348(11.4)$ & $3353(12.3)$ & $2279(14.3)$ \\
\hline Extremely stressful & $485(2.4)$ & $270(2.3)$ & $728(2.7)$ & $459(2.9)$ \\
\hline \multicolumn{5}{|l|}{ Self-rated health } \\
\hline Excellent & $3038(14.8)$ & $1692(14.3)$ & 4009 (14.7) & $2474(15.5)$ \\
\hline Very Good & $6241(30.4)$ & $3873(32.8)$ & 8467 (31.1) & $5532(34.7)$ \\
\hline Good & $6385(31.1)$ & $3771(32.0)$ & 8469 (31.1) & 4718 (29.6) \\
\hline Poor & $3376(16.5)$ & $1752(14.9)$ & 4521 (16.6) & $2275(14.3)$ \\
\hline Fair & $1442(7.0)$ & $685(5.8)$ & $1737(6.4)$ & $911(5.7)$ \\
\hline \multicolumn{5}{|l|}{ Health behaviours } \\
\hline
\end{tabular}




\section{Original research}

Table 2 Continued

\begin{tabular}{|c|c|c|c|c|}
\hline \multirow[b]{2}{*}{ Characteristic* } & \multicolumn{2}{|l|}{ Male cohort } & \multicolumn{2}{|l|}{ Female cohort } \\
\hline & Derivationt & Validation $\ddagger$ & Derivationt & Validation $\ddagger$ \\
\hline Current smoker & $3747(18.3)$ & $2527(21.4)$ & $11320(41.6)$ & $6396(40.2)$ \\
\hline Former smoker $\geq 5$ years & $9898(48.3)$ & $5340(45.3)$ & $7544(27.7)$ & $4758(29.9)$ \\
\hline Pack-years, median (IQR) & $19.5(7.2$ to 36.8$)$ & 18 (7 to 35$)$ & $11.2(5$ to 27$)$ & $11.0(5.0$ to 26.0$)$ \\
\hline Former smoker $<5$ years & $1351(6.6)$ & $627(5.3)$ & $1329(4.9)$ & $673(4.2)$ \\
\hline Non-smoker & $3305(16.1)$ & $1877(15.9)$ & $3972(14.6)$ & $2244(14.1)$ \\
\hline \multicolumn{5}{|l|}{ Alcohol } \\
\hline Current drinker & $16364(79.8)$ & $9436(80.0)$ & $18729(68.8)$ & $11200(70.3)$ \\
\hline Number of drinks last week, median (IQR) & 3 (0 to 8$)$ & $1(0$ to 4$)$ & 3 (0 to 9$)$ & $1(0$ to 4$)$ \\
\hline Former drinker§ & $3532(17.2)$ & $2319(19.7)$ & $6582(24.2)$ & $4682(29.4)$ \\
\hline Juice consumption, median (IQR) & $1.0(0.1$ to 1.0$)$ & $0.6(0.1$ to 1.0$)$ & $1.0(0.1$ to 1.0$)$ & $0.4(0.0$ to 1.0$)$ \\
\hline Potato consumption, median (IQR) & $0.4(0.3$ to 0.9$)$ & $0.4(0.1$ to 0.6$)$ & $0.4(0.1$ to 0.7$)$ & $0.3(0.1$ to 0.6$)$ \\
\hline \multicolumn{5}{|l|}{ Leisure physical activity } \\
\hline Daily energy expenditure (METs), median (IQR) & $1.5(0.5$ to 3.0$)$ & $1.6(0.6$ to 3.0$)$ & $1.1(0.3$ to 2.3$)$ & $1.2(0.4$ to 2.5$)$ \\
\hline \multicolumn{5}{|l|}{ Functional measures } \\
\hline \multicolumn{5}{|l|}{ Number of activities need help } \\
\hline None & $14272(69.6)$ & $10324(87.6)$ & $16460(60.4)$ & $12364(77.6)$ \\
\hline 1 & $816(4.0)$ & $642(5.4)$ & $2227(8.2)$ & $1637(10.3)$ \\
\hline 2 & $351(1.7)$ & $302(2.6)$ & $1143(4.2)$ & $827(5.2)$ \\
\hline 3 & $246(1.2)$ & $192(1.6)$ & $688(2.5)$ & $474(3.0)$ \\
\hline No & $16569(80.8)$ & $9613(81.5)$ & $23151(85.0)$ & $13891(87.2)$ \\
\hline \multicolumn{5}{|l|}{ Stroke } \\
\hline Yes & $751(3.7)$ & $382(3.2)$ & $859(3.2)$ & $471(3.0)$ \\
\hline No & $19741(96.3)$ & $11389(96.6)$ & $26351(96.8)$ & $15442(96.9)$ \\
\hline \multicolumn{5}{|l|}{ Diabetes } \\
\hline Yes & $3147(15.3)$ & $2203(18.7)$ & $3142(11.5)$ & $2120(13.3)$ \\
\hline No & $17341(84.6)$ & $9578(81.2)$ & $24078(88.4)$ & $13793(86.6)$ \\
\hline \multicolumn{5}{|l|}{ Mood disorder } \\
\hline Yes & $832(4.1)$ & $754(6.4)$ & $1816(6.7)$ & $1581(9.9)$ \\
\hline No & $15293(74.6)$ & $11020(93.5)$ & $19489(71.6)$ & $14335(90.0)$ \\
\hline \multicolumn{5}{|l|}{ High blood pressure } \\
\hline Yes & 7509 (36.6) & $5014(42.5)$ & $11533(42.3)$ & $7169(45.0)$ \\
\hline No & $12950(63.2)$ & $6751(57.3)$ & $15671(57.5)$ & $8737(54.8)$ \\
\hline \multicolumn{5}{|l|}{ COPD } \\
\hline Yes & $1432(7.0)$ & $774(6.6)$ & $2069(7.6)$ & $1344(8.4)$ \\
\hline No & $17430(85.0)$ & $10999(93.3)$ & $23035(84.6)$ & $14544(91.3)$ \\
\hline \multicolumn{5}{|l|}{ Epilepsy } \\
\hline Yes & $73(0.4)$ & $0(0)$ & $112(0.4)$ & $0(0)$ \\
\hline No & $14503(70.7)$ & $0(0)$ & $19505(71.6)$ & $0(0)$ \\
\hline Body mass index, median (IQR) & 26.4 (24.2 to 29.2 ) & 26.9 (24.4 to 29.7$)$ & 25.5 (22.8 to 29.0$)$ & 25.8 (23.0 to 29.6$)$ \\
\hline
\end{tabular}


Table 2 Continued

\begin{tabular}{|c|c|c|c|c|}
\hline \multirow[b]{2}{*}{ Characteristic* } & \multicolumn{2}{|l|}{ Male cohort } & \multicolumn{2}{|c|}{ Female cohort } \\
\hline & Derivationt & Validation‡ & Derivationt & Validation $\ddagger$ \\
\hline \multicolumn{5}{|l|}{ Survey year } \\
\hline 2001 & $4370(21.3)$ & & $5910(21.7)$ & \\
\hline 2003 & $5200(25.4)$ & & $6932(25.5)$ & \\
\hline 2005 & $5009(24.4)$ & & $6778(24.9)$ & \\
\hline $2007 / 2008$ & $5927(28.9)$ & & $7613(28.0)$ & \\
\hline $2009 / 2010$ & & $5801(49.2)$ & & 7848 (49.3) \\
\hline $2011 / 2012$ & & $5990(50.8)$ & & $8082(50.7)$ \\
\hline
\end{tabular}

${ }^{*} \mathrm{~N}$ and percentage unless otherwise specified; percentages will not sum to $100 \%$ due to missingness (see online supplemental table 2).

tCanadian Community Health Survey cycles conducted in 2000/2001, 2003, 2005 and 2007/2008.

¥Canadian Community Health Survey cycles conducted in 2009/2010 and 2011/2012.

$\S$ In CCHS 2007/2008, former drinkers can only be differentiated from non-drinkers in an optional module performed in only some regions; in 2009/2010 and 2011/2012, former drinkers cannot be differentiated from non-drinkers pulmonary disorder

CCHS, Canadian Community Health Surveys; COPD, chronic obstructive pulmonary disease; MET, metabolic equivalent of task.

Table 3 DemPoRT (Dementia Population Risk Tool) goodness of fit summary statistics of the full model in the derivation, validation and combined data, and the reduced model in the combined data*

\begin{tabular}{|c|c|c|c|c|}
\hline & Derivation & Validation & Combined & Reduced \\
\hline \multicolumn{5}{|l|}{ Male model } \\
\hline \multicolumn{5}{|l|}{ Discrimination } \\
\hline C-statistic $(95 \% \mathrm{Cl})$ & $0.82(0.80$ to 0.83$)$ & $0.83(0.81$ to 0.85$)$ & $0.82(0.81$ to 0.84$)$ & $0.82(0.81$ to 0.84$)$ \\
\hline Ratio of 95 to 5 risk percentile & $28.6(0.128 / 0.004)$ & $26.1(0.119 / 0.005)$ & $36.5(0.134 / 0.004)$ & $36.2(0.134 / 0.004)$ \\
\hline \multicolumn{5}{|l|}{ Calibration } \\
\hline Observed vs predicted ${ }^{1}$ & $-0.76 \%$ & $4.21 \%$ & $-0.61 \%$ & $-0.61 \%$ \\
\hline $\begin{array}{l}\text { 5-year cumulative incidence } \\
\text { (observed) }(95 \% \mathrm{Cl})\end{array}$ & $0.044(0.041$ to 0.047$)$ & 0.045 (0.041 to 0.048$)$ & $0.044(0.0420 .047)$ & $0.044(0.042$ to 0.047$)$ \\
\hline 5-year risk (predicted) & 0.045 & 0.043 & 0.045 & 0.045 \\
\hline Calibration slope and intercept & 0.7859 to 0.0098 & 0.7799 to 0.0080 & 0.8240 to 0.0081 & 0.8285 to 0.0079 \\
\hline \multicolumn{5}{|l|}{ Overall performance } \\
\hline Brier Score (scaled) & 0.081 & 0.068 & 0.086 & 0.086 \\
\hline Nagelkerke $\mathrm{R}^{2}$ & 0.105 & 0.123 & 0.100 & 0.100 \\
\hline \multicolumn{5}{|l|}{ Female model } \\
\hline \multicolumn{5}{|l|}{ Discrimination } \\
\hline C-statistic $(95 \% \mathrm{Cl})$ & 0.82 (0.81 to 0.83 ) & 0.83 (0.81 to 0.85$)$ & 0.83 (0.82 to 0.83 ) & 0.83 (0.82 to 0.83 ) \\
\hline Ratio of 95 to 5 risk percentile & $54.3(0.171 / 0.003)$ & $50.6(0.167 / 0.003)$ & $64.8(0.178 / 0.003)$ & $64.6(0.178 / 0.003)$ \\
\hline \multicolumn{5}{|l|}{ Calibration } \\
\hline Observed vs predicted* & $-1.07 \%$ & $-10.58 \%$ & $-0.78 \%$ & $-0.78 \%$ \\
\hline $\begin{array}{l}\text { 5-year cumulative incidence } \\
\text { (observed) }(95 \% \mathrm{Cl})\end{array}$ & 0.060 (0.057 to 0.062$)$ & $0.053(0.050$ to 0.057$)$ & $0.057(0.055$ to 0.060$)$ & $0.057(0.055$ to 0.060$)$ \\
\hline 5-year risk (predicted) & 0.060 & 0.059 & 0.058 & 0.058 \\
\hline Calibration slope and intercept & 0.7671 to 0.0145 & 0.8666 to 0.0128 & 0.8320 to 0.0102 & 0.8335 to 0.0101 \\
\hline \multicolumn{5}{|l|}{ Overall performance } \\
\hline Brier Score (scaled) & 0.107 & 0.102 & 0.111 & 0.111 \\
\hline Nagelkerke $\mathrm{R}^{2}$ & 0.147 & 0.132 & 0.133 & 0.133 \\
\hline
\end{tabular}

${ }^{*}$ Three types of performance tests were examined. ${ }^{22}$ (1) Discrimination is the ability of a predictive model to differentiate between those who experience the outcome from those who do not. C-statistic is a rank order statistic for predictions against true outcomes. ${ }^{24}$ The statistic ranges from 0 to 1 ; a value of 0.5 indicates the model is no better than random prediction, while a value of 1 indicates the model perfectly predicts whose who will develop the outcome of interest and who will not. Ratio of 95 to 5 risk percentile is a measure indicating the spread of the predicted risks, where a higher ratio indicates a more discriminating algorithm. For example, a ratio of 20 indicates that the absolute risk of the event of interest is 20 times higher for a person in the $95^{\text {th }}$ percentile of risk than for a person in the $5^{\text {th }}$ percentile of risk. (2) Calibration (or accuracy) describes how well the predicted probability of disease agrees with the observed outcomes. Observed versus predicted (O vs $P$ ) is the relative difference between the observed incidence and the predicted risk, calculated as (Observed - Predicted)/Observed $\times 100$. The absolute values for this calculation are the observed 5 -year cumulative incidence and the predicted 5 -year risk. A $1 \%$ difference indicates that $1 \%$ more events were observed than were predicted. This tables show overall 0 vs P. Online supplemental digital content 6 and 7 show 0 vs P for specific subgroups. The calibration slope and intercept indicates the slope and intercept of the calibration plots. Figure 1 displays the calibration plots for validation data. (3) Overall performance measures. Brier score (scaled) is a measure of overall agreement between observed and predicted risk with values between 0 and $1 .{ }^{33}$ Nagelkerke $R^{2}$ is a measure of the amount of variation in risk between individuals in the data that is explained by the model, with values from 0 to $1^{32}$. Larger values indicate that more variation is explained. 


\section{A)}
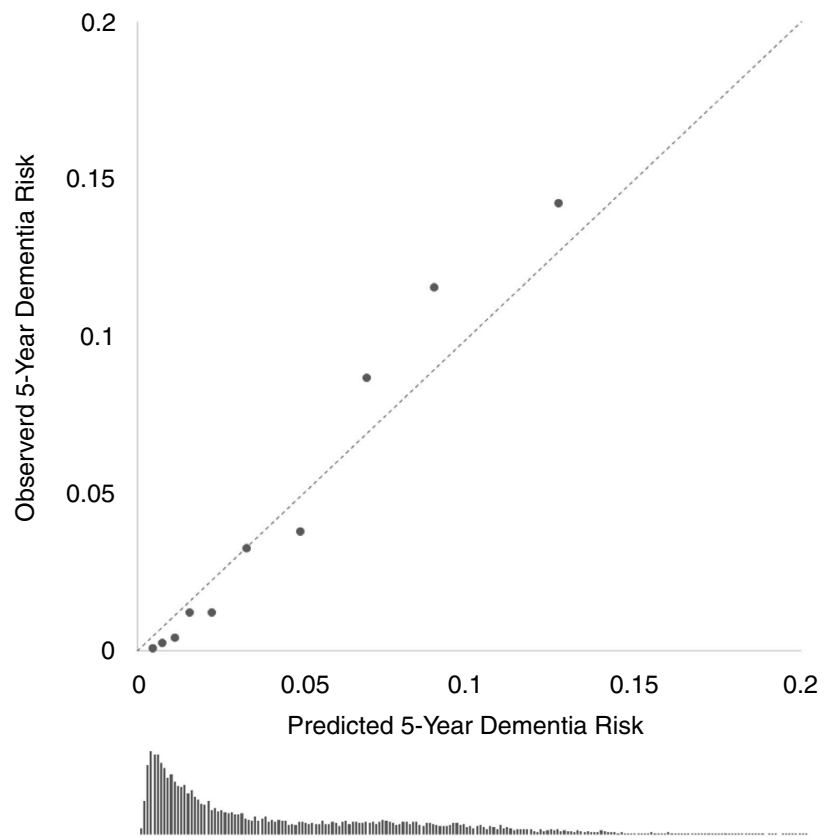

B)

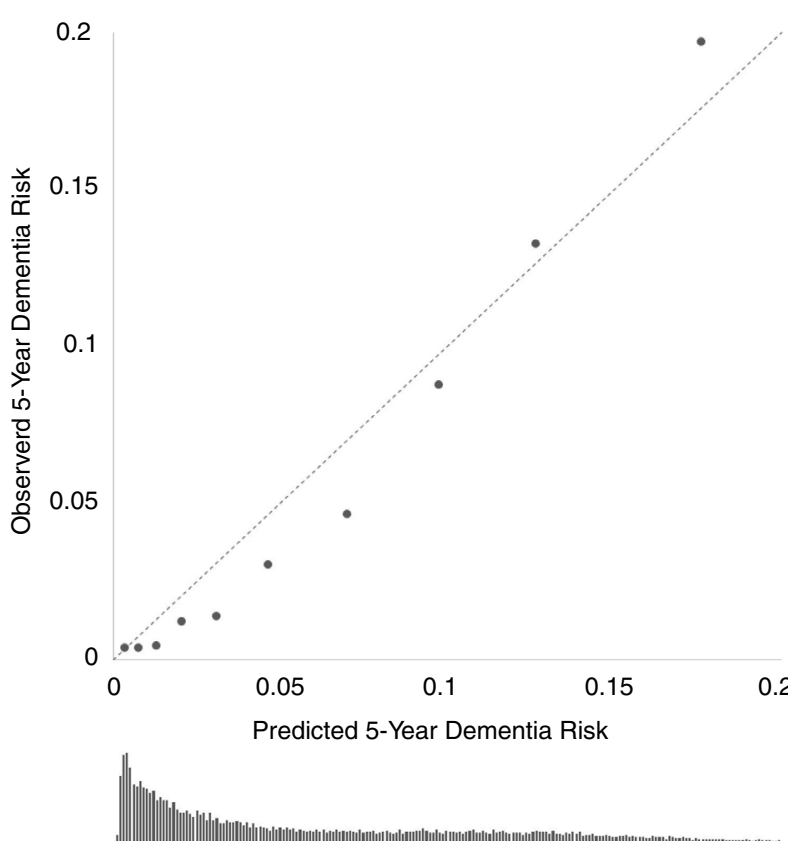

Figure 1 Calibration plots for the full model in validation data; mean predicted 5 -year risk of dementia versus observed dementia incidence for (A) men and (B) women, by decile of predicted risk. Histograms display the relative distribution of predicted risk in the population.

among men and 0.8666 among women. Among men, the model was well-calibrated in 68 of 88 predefined policy-relevant subgroups (online supplemental digital content 7 ), having no more than a $20 \%$ difference in predicted versus observed risk, evaluated among subgroups where at least $5 \%$ of individuals developed dementia. In women, the model was well-calibrated in 86 of 98 subgroups (online supplemental digital content 8). Both the male and female models underestimate dementia risk among those at older ages, those who need help with daily activities and who have a history of stroke. Well-calibrated subgroups include behavioural risk exposure categories, many socio-demographic groups, stress, self-rated health and by diabetes and hypertension status.

In models where the number of activities needing help and selfrated health variables are excluded, discriminative performance is slightly reduced in men (c-statistic: 0.81 ) and remains consistent in women (c-statistic: 0.83). Calibration by risk deciles and in population subgroups is not notably affected. Discriminative performance in the age alone models was slightly decreased when compared with the full models (male c-statistic: 0.80; female c-statistic: 0.81). Calibration across risk deciles was degraded in both the male and female age-alone models, especially among those at high risk. Calibration was also degraded across many subgroups of importance to policymakers, including ethnicity among men, current smokers, former drinkers and those with diabetes or a history of stroke.

\section{DISCUSSION}

The Dementia Population Risk Tool is a discriminating and wellcalibrated algorithm for predicting 5 -year incidence of dementia among community-dwelling individuals, developed using risk factor information relevant to population health and available to population health planners and decision makers. DemPoRT is a valuable tool for population health planning and policy development as it is discriminating and well-calibrated across many subgroups of importance to clinicians and policymakers. Inclusion of health behaviour variables facilitates the development and evaluation of primary prevention strategies, and inclusion of socio-demographic variables enables evaluation of dementia burden and prevention strategies with an equity perspective. ${ }^{26}$ As it was created using routinely collected population health survey data, DemPoRT can be easily applied to newer cycles of the CCHS or to other, similar national health surveys to produce up-to-date population-level estimates of dementia incidence. All variables were centred on their means, facilitating application of the models to new settings where some of the predictor variables are not available and by allowing for re-calibration to populations with different risk factor distributions.

Population-level evaluation of disease including the development of disease projections is best performed using multivariable predictive risk algorithms; ${ }^{7}$ the DemPoRT algorithm is uniquely suited for this application. A recent review identified 39 studies describing risk algorithms to predict dementia among those in late life. ${ }^{27}$ Sixteen studies described models with good discrimination (c-statistic of 0.80 to 0.89 ), while two studies described models with excellent discrimination (c-statistic: $0.91^{13}$ and $\left.0.93^{8}\right)$. Of these, all but one ${ }^{37}$ require neuropsychological $(n=16)$ or genetic $(n=4)$ testing; measures that are not available at the population level. Furthermore, most were developed in highly defined populations with small sample sizes and few dementia events, limiting their generalisability. Seven studies included any socio-demographic or lifestyle variables, most including only education $(n=5)$. The only well performing algorithm identified in this review that does not require neuropsychological or genetic testing and includes socio-demographic and lifestyle variables is the Dementia Risk Score (DRS), which predicts 5-year dementia risk among individuals 60 to 79 years of age using primary care data (c-statistic: 0.84$).{ }^{37}$ It includes measures for smoking (ascertained as current vs non-current smoker), heavy alcohol use (yes vs no), depression (yes vs no), social deprivation (quintiles) and aspirin use (yes vs no) in addition to various disease states. Linear age, age squared, linear body mass index (BMI) and BMI squared were included as continuous variables. The DRS may be useful for population health planning in the UK, as 
Box 1 Example of DemPoRT (Dementia Population Risk Tool) for individual use

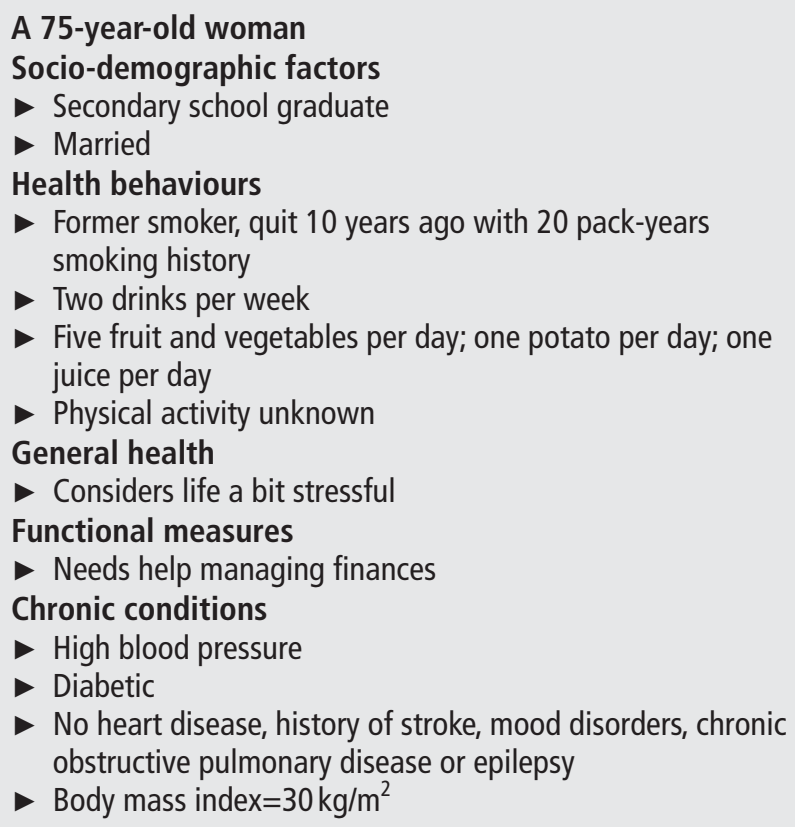

5 -year dementia risk $=9.7 \%$

it was developed using over 800000 patients from a nationally representative primary care database. However, development on clinical data limits generalisability and it lacks some of the sociodemographic and health behaviour variables (eg, education, ethnicity, diet, physical activity) that were found to be predictive of dementia in the present study. Potential usefulness for evaluating dementia prevention strategies is also limited by categorisation of continuous health behaviour variables, and calibration across subgroups relevant to population health planning has not be assessed. Existing dementia algorithms developed specifically for use in the general population have similar limitations. ${ }^{38}$

DemPoRT can also be used by individual patients or their clinicians to assess dementia risk and inform decisions about lifestyle modification. See box 1 for an example of DemPoRT for individual use. As all variables are self-reported, dementia risk can be evaluated both within and outside of the clinical setting; an online calculator to facilitate this use is available at https:// www.projectbiglife.ca/. Variable centering enables risk assessment in individuals who provide only partial responses and allow for dynamic risk calculation as an individual completes the questionnaire, providing a more interactive and engaging experience. Our team has developed online health calculators as knowledge translation tools for other risk algorithms, including for cardiovascular disease, which was developed using the same health survey data as DemPoRT. ${ }^{19}$ The cardiovascular disease risk tool is integrated into the Heart and Stroke Foundation of Canada website to provide individualised risk calculations for their eHealth Risk Assessment programme. Knowledge translation of DemPoRT is facilitated by this online tool, in addition to the numerous online files.

Most predictive algorithms for dementia that include only socio-demographic and health behaviour variables perform poorly. ${ }^{27}$ We attribute the favourable performance of DemPoRT to greater model complexity including the use of more variables, interaction terms and flexible functions for continuous variables.
Predictive model development generally prioritises simplicity and parsimony with the goal of producing algorithms that are easier to interpret and use. More complex algorithms do not have to be more burdensome in their application, however, particularity if they are implemented as reflexive online tools and if unbiassed calculations can be performed with partial responses. Overfitting is also often associated with increased model complexity. Our approach to model pre-specification limited this risk in the development of the DemPoRT models, which performed well in validation. While increasing model complexity may be considered unnecessary due to the generally marginal increase in overall discrimination with the addition of risk factors beyond the most predictive, added complexity has the potential to improve model discrimination for individuals and population subgroups. Complex algorithms like DemPoRT therefore have potential to support both clinical and populationbased precision medicine. ${ }^{19}$

Favourable performance of DemPoRT may also be due in part to the choice of modelling technique. Given the late-life onset of dementia, it is important to consider the competing risk of death when developing a dementia risk prediction model, as failure to do so can result in risk overestimation. ${ }^{39}$ Although Cox proportional hazard modelling can be used, it has been suggested that Fine and Gray subdistribution hazard models, which model the subdistribution hazard function rather than the hazard function, are better suited to prediction purposes. ${ }^{28}$ We are only aware of one other dementia model that has used Fine and Gray regression. ${ }^{15}$

\section{Limitations}

One concern with complex prediction models is an increased risk of overfitting, which can lead to the algorithm performing poorly in external populations despite performing well in internal or temporal validation. The DemPoRT model is unlikely to be overfitted for several reasons. First, the model was fully prespecified, ${ }^{18}$ which limits the potential for overfitting by avoiding bias introduced by data-driven variable selection procedures. ${ }^{24}$ DemPoRT was also developed on a very large data set with more than enough sample size for the prespecified df, and there was no evidence for overfitting in the full model. Lastly, other algorithms developed in Ontario using similar data and variable specifications have been successfully validated in external populations. A diabetes risk algorithm was validated in Manitoba, another Canadian province; discrimination was slightly improved and predicted risk closely approximated observed risk after recalibration ${ }^{40}$ and an algorithm for all-cause mortality developed using the Ontario CCHS was successfully validated using national CCHS data. ${ }^{26}$

As the case ascertainment algorithm is imperfect, and only ascertains physician-diagnosed dementia, some individuals with dementia are not being identified. Dementia is known to be generally underdiagnosed; ${ }^{41}{ }^{42}$ individuals with less severe dementia symptoms, those who have significant home supports, or have poor access to healthcare may be missed or identified later in the disease trajectory. DemPoRT's performance may therefore be overestimated, although overall performance and performance across population subgroups remains acceptable. Like the previously mentioned DRS model developed using routinely-available primary care data, ${ }^{37}$ DemPoRT also underestimated dementia risk at the oldest ages-likely due to the unavailability of variables with additional predictive ability among these adults. Other models have had success developing algorithms for older adults using neurophysiological testing. ${ }^{911}$ 
That said, DemPoRT's underestimation can be corrected for in dementia projections using age and sex-specific recalibration techniques. $^{43}$

Other population dementia models have included variables for traumatic brain injury, cholesterol, cognitively stimulating activities and fish consumption, ${ }^{38}$ which may improve model performance. Additionally, some surveys, like the Scottish Health Survey, ascertain health behaviour information using more detailed and standardised measures than the CCHS, ${ }^{44}$ however the current specification is much more common in health surveys worldwide. As DemPoRT uses self-reported predictor information ascertained at baseline, model performance may also be improved with more accurate, longitudinal predictor assessment-however, model performance was favourable regardless and the use of self-report data enhances application potential. As long as variables are ascertained similarly and reporting patterns do not change, model performance in application is unlikely to be affected by the use of self-reported data.

\section{Conclusion}

DemPoRT is the first multivariable predictive risk algorithm for dementia designed specifically for use by population health planners, with favourable performance despite using only selfreported population-level data and without the use of neuropsychological or genetic testing. It is discriminating and able to predict dementia risk across a range of health profiles. DemPoRT will be used to answer key policy questions with respect to the future burden of dementia in Canada and will support the development and evaluation of population-level dementia prevention strategies.

\section{What is already known on this subject}

- Most predictive algorithms for dementia risk have been designed for use in the clinical context, and none have been developed for population health planning purposes. Additionally, most algorithms for dementia that include only socio-demographic and health behaviour variables perform poorly.

\section{What this study adds}

- The Dementia Population Risk Tool (DemPoRT) is discriminating and well-calibrated across a wide range of population subgroups despite using only self-reported risk factors. Favourable performance is attributed to modelling technique and greater model complexity including the use of more variables, interaction terms and flexible functions for continuous variables. DemPoRT is the first multivariable risk prediction algorithm for dementia designed for population use. This algorithm will be used to produce improved estimates of future dementia burden, identify high risk population subgroups and inform the development of dementia prevention strategies. It can also be patients and their clinicians to assess individual dementia risk.

\section{Twitter Stacey Fisher @StaceyFisher_and Amy T Hsu @amytmhsu}

Contributors SF was responsible for the study design, protocol development, data analysis, interpretation of the results and drafting and revision of the manuscript. DGM and PT were responsible for conception of the project, grant application and contributed to the study design, protocol development and result interpretation. MTu, ABE and MTa contributed to the study design, protocol development and result interpretation and provided data/statistical support. CB, MJ and ATH contributed to the design of the study, protocol development and result interpretation. YS provided statistical support and is primarily responsible for the online web calculator, visualisation tool and application programming interface. GA contributed to result interpretation. All authors provided critical reviews of the manuscript.

Funding The results reported herein correspond to specific aims of grant MOP 142237 to Douglas G Manuel from the Canadian Institutes of Health Research (CIHR). This study was supported by ICES, formerly known as the Institute of Clinical Evaluative Sciences, which is funded by an annual grant from the Ontario Ministry of Health and Long-Term Care (MOHLTC). The opinions, results and conclusions reported in this paper are those of the authors and are independent from the funding sources. No endorsement by CIHR, ICES or the Ontario MOHLTC is intended or should be inferred.

\section{Competing interests None declared.}

\section{Patient consent for publication Not required.}

Data availability statement Data were linked using unique encoded identifiers and analysed at ICES. The data set from this study is held securely in coded form at ICES. While data sharing agreements prohibit ICES from making the data set publicly available, access may be granted to those who meet prespecified criteria for confidential access, available at www.ices.on.ca/DAS. The full data set creation plan and underlying analytical code are available from the authors upon request, understanding that the programmes may rely upon coding templates or macros that are unique to ICES and are therefore either inaccessible or may require modification.

Supplemental material This content has been supplied by the author(s). It has not been vetted by BMJ Publishing Group Limited (BMJ) and may not have been peer-reviewed. Any opinions or recommendations discussed are solely those of the author(s) and are not endorsed by BMJ. BMJ disclaims all liability and responsibility arising from any reliance placed on the content. Where the content includes any translated material, BMJ does not warrant the accuracy and reliability of the translations (including but not limited to local regulations, clinical guidelines, terminology, drug names and drug dosages), and is not responsible for any error and/or omissions arising from translation and adaptation or otherwise.

Open access This is an open access article distributed in accordance with the Creative Commons Attribution Non Commercial (CC BY-NC 4.0) license, which permits others to distribute, remix, adapt, build upon this work non-commercially, and license their derivative works on different terms, provided the original work is properly cited, appropriate credit is given, any changes made indicated, and the use is non-commercial. See: http://creativecommons.org/licenses/by-nc/4.0/.

\section{ORCID iDs}

Stacey Fisher http://orcid.org/0000-0002-4925-7806

Douglas G Manuel http://orcid.org/0000-0003-0912-0845

\section{REFERENCES}

1 Alzheimer's Disease International. World Alzheimer Report 2018 - The State of the Art of Dementia Research: New Frontiers. London, UK, 2018.

2 Norton S, Matthews FE, Barnes DE, et al. Potential for primary prevention of Alzheimer's disease: an analysis of population-based data. Lancet Neurol 2014:13:788-94.

3 Public Health Agency of Canada. A dementia strategy for Canada: together we Aspire. Ottawa, ON, 2019.

4 World Health Organization. Dementia: a public health priority. Geneva, Switzerland, 2012.

5 Australian Health Ministers Advisory Council. National framework for action on dementia 2015-2019. Canberra, Australia: Deparment of Health, 2015.

6 Assistant Secretary for Planning and Evaluation U.S. Department of Health and Human Services. National Plans to Address Alzheimer's Disease. Washington, D.C, 2012.

7 Manuel DG, Rosella LC, Hennessy D, et al. Predictive risk algorithms in a population setting: an overview. J Epidemiol Community Health 2012;66:859-65.

8 Mura T, Baramova M, Gabelle A, et al. Predicting dementia using socio-demographic characteristics and the free and cued selective reminding test in the general population. Alzheimers Res Ther 2017;9:21.

9 Wolfsgruber $\mathrm{S}$, Jessen F, Wiese B, et al. The CERAD neuropsychological assessment battery total score detects and predicts Alzheimer disease dementia with high diagnostic accuracy. Am J Geriatr Psychiatry 2014;22:1017-28.

10 Barnes DE, Covinsky KE, Whitmer RA, et al. Predicting risk of dementia in older adults: the late-life dementia risk index. Neurology 2009;73:173-9.

11 Derby CA, Burns LC, Wang C, et al. Screening for predementia AD: time-dependent operating characteristics of episodic memory tests. Neurology 2013;80:1307-14.

12 Meng X, D'Arcy C, Morgan D, et al. Predicting the risk of dementia among Canadian seniors: a useable practice-friendly diagnostic algorithm. Alzheimer Dis Assoc Disord 2013;27:23-9. 
13 Jungwirth S, Zehetmayer S, Bauer $\mathrm{P}$, et al. Prediction of Alzheimer dementia with short neuropsychological instruments. J Neural Transm 2009;116:1513-21.

14 Reitz C, Tang M-X, Schupf N, et al. A summary risk score for the prediction of Alzheimer disease in elderly persons. Arch Neurol 2010;67:835-41.

15 Licher S, Leening MJG, Yilmaz P, et al. Development and validation of a dementia risk prediction model in the general population: an analysis of three longitudinal studies. Am J Psychiatry 2019;176:543-51.

16 Kivipelto M, Ngandu T, Laatikainen T, et al. Risk score for the prediction of dementia risk in 20 years among middle aged people: a longitudinal, population-based study. Lancet Neurol 2006;5:735-41.

17 Anstey KJ, Cherbuin N, Herath PM. Development of a New Method for Assessing Global Risk of Alzheimer's Disease for Use in Population Health Approaches to Prevention. Prev Med 2013:14:411-21.

18 Fisher S, Hsu A, Mojaverian N, et al. Dementia population risk tool (DemPoRT): study protocol for a predictive algorithm assessing dementia risk in the community. BMJ Open 2017;7:e018018.

19 Manuel DG, Tuna M, Bennett C, et al. Development and validation of a cardiovascular disease risk-prediction model using population health surveys: the cardiovascular disease population risk tool (CVDPoRT). CMAJ 2018:190:E871-82.

20 Collins GS, Reitsma JB, Altman DG, et al. Transparent reporting of a multivariable prediction model for individual prognosis or diagnosis (TRIPOD): the TRIPOD statement. Eur Urol 2015;67:1142-51.

21 Béland Y. Canadian community health survey--methodological overview. Health Rep 2002;13:9-14.

22 Moons KGM, Altman DG, Reitsma JB, et al. Transparent reporting of a multivariable prediction model for individual prognosis or diagnosis (TRIPOD): explanation and elaboration. Ann Intern Med 2015;162:W1-73.

23 Jaakkimainen RL, Bronskill SE, Tierney MC, et al. Identification of Physician-Diagnosed Alzheimer's Disease and Related Dementias in Population-Based Administrative Data: A Validation Study Using Family Physicians' Electronic Medical Records. JAD 2016:54:337-49.

24 Harrell FE. Regression modeling strategies with Applicaitons to linear models, logistic regression and survival analysis. New York: Springer, 2001.

25 Steyerberg EW. Clinical prediction models. New York: Springer, 2009.

26 Manuel DG, Perez R, Sanmartin C, et al. Measuring burden of unhealthy behaviours using a multivariable predictive approach: life expectancy lost in Canada attributable to smoking, alcohol, physical inactivity, and diet. PLoS Med 2016;13:e1002082-27.

27 Hou X-H, Feng L, Zhang C, et al. Models for predicting risk of dementia: a systematic review. J Neurol Neurosurg Psychiatry 2019;90:373-9.
28 Fine JP, Gray RJ. A proportional hazards model for the Subdistribution of a competing risk. J Am Stat Assoc 1999:94:496-509.

29 Van Houwelingen JC, Le Cessie S. Predictive value of statistical models. Stat Med 1990;9:1303-25.

30 Gelman A. Struggles with survey weighting and regression modeling. Statist. Sci 2007:22:153-64.

31 Ambler G, Brady AR, Royston P. Simplifying a prognostic model: a simulation study based on clinical data. Stat Med 2002;21:3803-22.

32 Nagelkerke NJD. A note on a general definition of the coefficient of determination. Biometrika 1991;78:691-2.

33 Rufibach K. Use of Brier score to assess binary predictions. J Clin Epidemiol 2010;63:938-9.

34 R Core Team. R: a language and environment for statistical computing, 2016.

35 Gerds TA, Blanche P, Mogense UB, et al. Risk regression: risk regression models and prediction scores for Survial analysis with competing risks, 2020.

36 Harrell FE. Hmisc: Harrell miscellaneous, 2020.

37 Walters K, Hardoon S, Petersen I, et al. Predicting dementia risk in primary care: development and validation of the dementia risk score using routinely collected data. BMC Med 2016:14:1-12.

38 Licher S, Yilmaz P, Leening MJG, et al. External validation of four dementia prediction models for use in the general community-dwelling population: a comparative analysis from the Rotterdam study. Eur J Epidemio/ 2018;33:645-55.

39 Koller MT, Raatz H, Steyerberg EW, et al. Competing risks and the clinical community: irrelevance or ignorance? Stat Med 2012:31:1089-97.

40 Rosella LC, Manuel DG, Burchill C, et al. A population-based risk algorithm for the development of diabetes: development and validation of the diabetes population risk tool (DPoRT). J Epidemiol Community Heal 2011;65:613-20.

41 Connolly A, Gaehl E, Martin H, et al. Underdiagnosis of dementia in primary care: variations in the observed prevalence and comparisons to the expected prevalence. Aging Ment Health 2011:15:978-84.

42 Kosteniuk JG, Morgan DG, O'Connell ME, et al. Incidence and prevalence of dementia in linked administrative health data in Saskatchewan, Canada: a retrospective cohort study. BMC Geriatr 2015;15:73.

43 Vergouwe $Y$, Nieboer D, Oostenbrink R, et al. A closed testing procedure to select an appropriate method for updating prediction models. Stat Med 2017;36:4529-39

44 Fisher S, Bennett C, Hennessy D, et al. International population-based health surveys linked to outcome data: a new resource for public health and epidemiology. Health Rep 2020;31:12-23. 